\title{
Analytical Calculation of Differential and Integral Electromagnetic Characteristics of MHD-Stirrer
}

\author{
Viktor N. Timofeev and Maksim Yu. Khatsyuk* \\ Siberian Federal University \\ 79 Svobodny, Krasnoyarsk, 660041, Russia
}

Received 14.11.2016, received in revised form 26.12.2016, accepted 19.01.2017

The analysis of electromagnetic field in the system «inductor - bath of molten (liquid metal)" by different winding characteristics of the inductor due to analytical method is shown in this paper. Integral and differential characteristics of the system are defined and compared for the most common coil inductors MHD-stirrers. It provides a framework for the analysis of field harmonics on the surface of melt for various types of inductors. Also made the comparative assessment of influence of longitudinal edge effect on system performance.

Keywords: MHD-stirrers, electromagnetic characteristics, analytical calculation.

\section{Аналитический расчет дифференциальных \\ и интегралыных электромагнитных характеристик \\ МГД-перемешивателя}

\author{
В.Н. Тимофеев, М.Ю. Хацаюк \\ Сибирский федеральный университет \\ Россия, 660041, Красноярск, пр. Свободный, 79
}

\begin{abstract}
В данной статье аналитическим методом проведен анализ электромагнитного поля в системе «индуктор - ванна с расплавом (жидкий металл)» при различных обмоточных характеристиках индуктора. Определены интегральные и дифференциильные характеристики системы для наиболее распространенных обмоток индукторов МГД-перемешивателя и проведено их сравнение. Представлена основа для анализа гармонического состава поля на поверхности расплава для различных типов индукторов. Также проведена сравнительная оценка влияния продольного краевого эффекта на показатели системы.
\end{abstract}

(c) Siberian Federal University. All rights reserved

* Corresponding author E-mail address: maxhac@ya.ru, viktortim0807@mail.ru 
Ключевые слова: МГД-перемешиватель, электромагнитная характеристика, аналитический расчет

\section{Введение}

В процессе приготовления многокомпонентных сплавов важной технологической операцией является перемешивание расплава с целью выравнивания химического состава и температуры во всем объеме ванны миксера. Перемешивание расплава в плавильных печах позволяет повысить интенсивность плавления и сократить на эту операцию потребление энергии. Основным достоинством электромагнитного перемешивания признано отсутствие контакта с высокотемпературным и агрессивным расплавом и возможность автоматизировать операцию перемешивания. В металлургической практике наибольшее распространение получили индукционные магнитогидродинамические (МГД) перемешиватели, воздействующие на расплав бегущим магнитным полем. Источником бегущего магнитного поля служит индуктор, представляющий собой катушки, расположенные на магнитопроводе, сдвинутые в пространстве и питаемые $m$-фазной системой переменных токов. Как правило, электромагнитные, тепловые и гидродинамические процессы в миксерах с МГД-перемешивателями моделируются численными методами с использованием таких программных пакетов, как ANSYS и т.д. [1] На ранней стадии проектирования МГД-перемешивателей требуется выбрать тип обмотки индуктора, количество фаз, частоту питающего напряжения, токовую нагрузку и т.д. Для этого требуется простая методика проектирования этих устройств. В [2] решена одномерная задача анализа электромагнитных процессов в МГД-перемешивателе. Интегральные и дифференциальные характеристики получены при наличии только первой гармоники электромагнитного поля в зазоре. В данной статье аналитически решена двухмерная задача по анализу электромагнитного поля в МГД-перемешивателе с применением рядов Фурье в комплексной форме. Полученные выражения позволяют учитывать тип обмотки, количество и размеры пазов, распределение токовой нагрузки и влияние продольного краевого эффекта.

\section{Расчетная модель системы «МГД-перемешиватель - ванна с расплавом»}

Эскиз системы, состоящей из ванны с жидким металлом (расплавом) и индуктора бегущего магнитного поля, представлен на рис. 1.

Индуктор расположен на расстоянии $\delta$ от расплава и имеет длину $L=2 p \tau$, где $p$ - число пар полюсов; $\tau$ - полюсное деление.

Для анализа электромагнитного поля примем следующие допущения:

1. Магнитопровод индуктора имеет магнитную проницаемость $\mu=\infty$ и электропроводность $\gamma=0$. В $M$ пазов магнитопровода уложены проводники с током, каждый $m$-й паз заменен поверхностным током с комплексной линейной плотностью

$$
\dot{J}_{m}=\frac{w_{m} \dot{I}_{m}}{2 \Delta_{m}}
$$

где $w_{m}$ - число проводников; $\dot{I}_{m}-$ комплексный ток; $2 \Delta_{m}-$ ширина $m$-го паза.

2. Воздушный зазор равномерный и равен 


$$
\delta^{\prime}=k_{\delta} k_{m} \delta,
$$

где $k_{\delta}$ и $k_{m}$ - коэффициенты, учитывающие влияния пазов и магнитного сопротивления ярма и зубцов соответственно.

3. Система имеет бесконечную длину в направлении оси $y$, т.е. электромагнитное поле является плоскопараллельным. Вектор комплексной напряженности электрического поля $\dot{\bar{E}}=\bar{e}_{y} \cdot \dot{E}_{y}$, вектор комплексной напряженности магнитного поля $\dot{\bar{H}}=\bar{e}_{x} \cdot \dot{H}_{x}+\bar{e}_{z} \cdot \dot{H}_{z}$, где $\bar{e}_{x}, \bar{e}_{y}, \bar{e}_{z}-$ единичные векторы прямоугольной системы координат.

4. Систему координат считаем жестко связанной с индуктором, а все элементы металла в ванне двигаются относительно индуктора с одинаковой скоростью $u$.

При принятых допущениях расчетная модель системы будет иметь вид, представленный на рис. 2.

\section{Дифференциальные уравнения и граничные условия}

Решение будем искать для комплексной напряженности электрического поля в области $1\left(0 \leq z \leq \delta^{\prime}\right)$ и области $2\left(\delta^{\prime}<z<\infty\right) \dot{\bar{E}}_{1}=\bar{e}_{y} \cdot \dot{E}_{1 y} ; \dot{\bar{E}}_{2}=\bar{e}_{y} \cdot \dot{E}_{2 y}$.

В расчетных областях справедливы дифференциальные уравнения и граничные условия [3]:

$$
\frac{\partial^{2} \dot{E}_{1 y}}{\partial z^{2}}+\frac{\partial^{2} \dot{E}_{1 y}}{\partial x^{2}}=0 ; \quad \frac{\partial^{2} \dot{E}_{2 y}}{\partial z^{2}}+\frac{\partial^{2} \dot{E}_{2 y}}{\partial x^{2}}-i \cdot \mu_{0} \cdot \gamma \cdot \omega \cdot \dot{E}_{2 y}-\mu_{0} \cdot \gamma \cdot u \frac{\partial \dot{E}_{2 y}}{\partial x}=0
$$

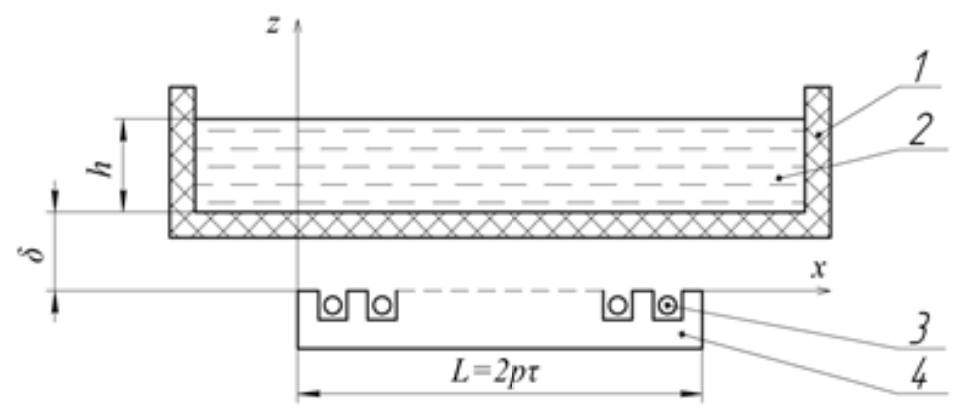

Рис. 1. Индуктор бегущего магнитного поля вблизи ванны с расплавом: 1 - прямоугольная ванна; 2 - расплав; 3 - многофазная обмотка; 4 - магнитопровод индуктора

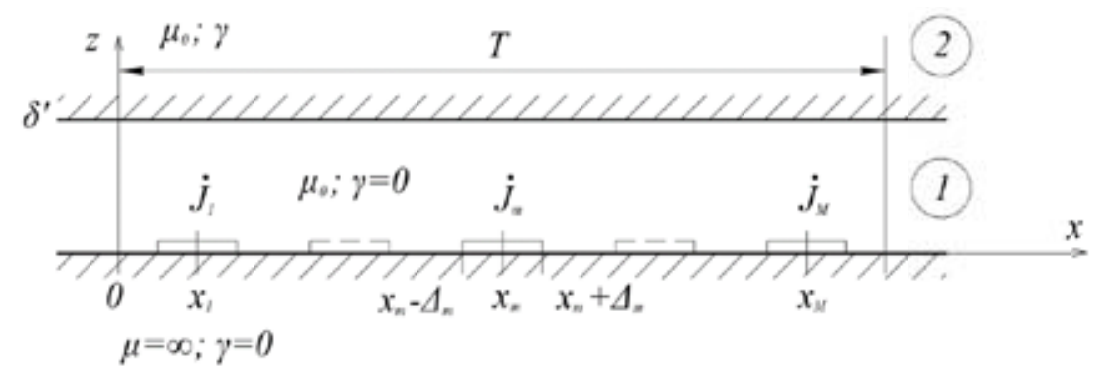

Рис. 2. Расчетная модель МГД-перемешивателя расплава в ванне, $x_{m}$ - расстояние до центра $m$-го паза от начала системы координат 


$$
\begin{aligned}
& \left.\frac{\partial \dot{E}_{1 y}}{\partial z}\right|_{z=0}=i \cdot \omega \cdot \mu_{0}\left\{\begin{array}{c}
\dot{J}_{m} ; x_{m}-\Delta_{m} \prec x \prec x_{m}+\Delta_{m} \\
0,
\end{array}\right. \\
& \left.\dot{E}_{1 y}\right|_{z=\delta^{\prime}}=\left.\dot{E}_{2 y}\right|_{z=\delta^{\prime}} ;\left.\quad \frac{\partial \dot{E}_{1 y}}{\partial z}\right|_{z=\delta^{\prime}}=\left.\frac{\partial \dot{E}_{2 y}}{\partial z}\right|_{z=\delta^{\prime}} ;\left.\quad \dot{E}_{2 y}\right|_{z \rightarrow \infty}=0 .
\end{aligned}
$$

Здесь $i=\sqrt{-1} ; \mu_{0}=4 \cdot \pi \cdot 10^{-7} \Gamma \mathrm{H} / \mathrm{M} ; \omega=2 \cdot \pi \cdot f \mathrm{c}^{-1} ; f$ - частота тока, Гц; $\gamma-$ удельная электропроводность расплава, $1 /($ Ом'м).

Комплексная линейная плотность тока $\dot{J}_{m}$ определяется выражением (2), где

$$
\dot{J}_{m}=J_{m} e^{i \varphi_{m}} .
$$

Величина фазы $m$-го тока зависит от количества фаз, числа пар полюсов $p$ и количества пазов $M$.

\section{Решение дифференциальных уравнений}

Полагая, что электромагнитное поле периодично по координате $x$ с периодом $T$, будем искать решения в виде рядов Фурье в комплексной форме [4]:

$$
\begin{aligned}
& \dot{E}_{1,2}(x, z)=\sum_{n=-\infty}^{+\infty} \dot{E}_{1,2 n}(z) e^{i k_{n} x}, \\
& \dot{E}_{1,2 n}(z)=\frac{1}{T} \int_{0}^{T} \dot{E}_{1,2}(x, z) e^{-i k_{n} x} \partial x ; \\
& k_{n}=\frac{2 n \pi}{T} .
\end{aligned}
$$

Умножив уравнения (3) и (4) на функцию $e^{-i k_{n} x}$ и проинтегрировав получившееся выражение по $x$ от 0 до $T$, получим:

$$
\begin{aligned}
& \frac{d^{2} \dot{E}_{1 n}}{d z^{2}}-k_{n}{ }^{2} \dot{E}_{1 n}=0 ; \\
& \frac{d^{2} \dot{E}_{2 n}}{d z^{2}}-\varphi_{n}{ }^{2} \dot{E}_{2 n}=0 ; \\
& \varphi_{n}{ }^{2}=k_{n}{ }^{2}+i \cdot \mu_{0} \cdot \gamma \cdot \omega+i \cdot k_{n} \cdot \mu_{0} \cdot \gamma \cdot u .
\end{aligned}
$$

Общие решения обыкновенных дифференциальных уравнений (12) и (13) определяются выражениями

$$
\dot{E}_{1 n}=A_{1} e^{k_{n} z}+A_{2} e^{-k_{n} z} ; \quad \dot{E}_{2 n}=A_{3} e^{\varphi_{n} z}+A_{4} e^{-\varphi_{n} z}
$$

Постоянные интегрирования $A_{j}(j=1 . .4)$ определяются из граничных условий (3-5), которые также необходимо преобразовать в соответствии с (10). При этом условие (3) заменяется выражением

$$
\left.\frac{d \dot{E}_{1 n}}{d z}\right|_{z=0}=\psi_{n},
$$


где

$$
\psi_{n}=\frac{i \omega \mu_{0}}{T} \sum_{m=1}^{M} \dot{J}_{m} \int_{x_{m}-\Delta_{m}}^{x_{m}+\Delta_{m}} e^{-i k_{n} x} \partial x=i \frac{2 \omega \mu_{0}}{k_{n} T} \sum_{m=1}^{M} \dot{J}_{m} \sin \left(k_{n} \Delta_{m}\right) e^{-i k_{n} x_{m}},
$$

а условия (4) и (5) сохраняются для $\dot{E}_{1 n}$ и $\dot{E}_{2 n}$.

Определив постоянные интегрирования из граничных условий и подставив их в (15) и (16), получим:

где

$$
\dot{E}_{1 n}=-\frac{\psi_{n}}{k_{n}} \frac{G_{n}(z)}{Q_{n}} ; \quad \dot{E}_{2 n}=-\psi_{n} \frac{e^{\varphi_{n}(\delta-z)}}{Q_{n}}
$$

$$
\begin{aligned}
& G_{n}(z)=k_{n} \cosh \left(k_{n}(z-\delta)\right)-\varphi_{n} \sinh \left(k_{n}(z-\delta)\right) \\
& Q_{n}=k_{n} \sinh \left(k_{n} \delta\right)+\varphi_{n} \cosh \left(k_{n} \delta\right) .
\end{aligned}
$$

В соответствии с (6) запишем искомые решения:

$$
\begin{aligned}
& \dot{E}_{1 y}(x, z)=-i \frac{2 \omega \mu_{0}}{T} \sum_{n=-\infty}^{\infty} \frac{1}{k_{n}^{2}} \frac{G_{n}(z)}{Q_{n}} C_{1 n} e^{i k_{n} x} \\
& \dot{E}_{2 y}(x, z)=-i \frac{2 \omega \mu_{0}}{T} \sum_{n=-\infty}^{\infty} \frac{1}{k_{n}} \frac{e^{\varphi_{n}(\delta-z)}}{Q_{n}} C_{1 n} e^{i k_{n} x}
\end{aligned}
$$

где

$$
C_{1 n}=\sum_{m=1}^{M} \dot{J}_{m} \sin \left(k_{n} \Delta_{m}\right) e^{-i k_{n} x_{m}}
$$

Из уравнения Максвелла $\operatorname{rot} \mathrm{E}=-i \omega \mathrm{B}$, имеем:

$$
\dot{B}_{x}=\frac{1}{i \omega} \frac{\partial \dot{E}_{y}}{\partial z} ; \quad \dot{B}_{z}=\frac{1}{i \omega} \frac{\partial \dot{E}_{y}}{\partial x}
$$

Подставив (23) и (24) в (26), получим:

$$
\begin{array}{ll}
\dot{B}_{x 1}=-\frac{2 \mu_{0}}{T} \sum_{n=-\infty}^{\infty} \frac{1}{k_{n}} \frac{G_{n}^{\prime}(z)}{Q_{n}} C_{1 n} e^{i k_{n} x} ; & \dot{B}_{z 1}=i \frac{2 \mu_{0}}{T} \sum_{n=-\infty}^{\infty} \frac{1}{k_{n}} \frac{G_{n}(z)}{Q_{n}} C_{1 n} e^{i k_{n} x} ; \\
\dot{B}_{x 2}=\frac{2 \mu_{0}}{T} \sum_{n=-\infty}^{\infty} \frac{\varphi_{n}}{k_{n}} \frac{e^{\varphi_{n}(\delta-z)}}{Q_{n}} C_{1 n} e^{i k_{n} x} ; & \dot{B}_{z 2}=i \frac{2 \mu_{0}}{T} \sum_{n=-\infty}^{\infty} \frac{1}{k_{n}} \frac{e^{\varphi_{n}(\delta-z)}}{Q_{n}} C_{1 n} e^{i k_{n} x}
\end{array}
$$

где

$$
G_{n}^{\prime}(z)=k_{n} \sinh \left(k_{n}(z-\delta)\right)-\varphi_{n} \cosh \left(k_{n}(z-\delta)\right) .
$$

Плотность тока в области 2 определяется законом Ома с учетом движения расплава со скоростью $и$ относительно системы координат:

$$
\begin{aligned}
& \dot{\delta}_{2 y}=\gamma\left(\dot{E}_{2}+u \dot{B}_{2 z}\right)=i \frac{2 \pi}{T \tau} \sum_{n=-\infty}^{\infty} \frac{e^{\varphi_{n}(\delta-z)}}{n Q_{n}} C_{1 n} \beta(s) e^{i k_{n} x} \\
& \beta(s)=\varepsilon\left(n(1-s)-\frac{T^{*}}{2}\right)
\end{aligned}
$$


где $\varepsilon=\omega \gamma \mu_{0} \tau^{2} / \pi^{2} ; T^{*}=T / \tau$.

\section{Интегральные и дифференциальные характеристики МГД-перемешивателя}

Комплексная электромагнитная мощность, отдаваемая обмоткой индуктора в зазор, равна

$$
\dot{S}_{e m}=-l_{\delta} \sum_{m=1}^{M} \int_{x_{m}-\Delta_{m}}^{x_{m}+\Delta_{m}} \dot{E}_{1}(x, 0) \cdot J_{m}^{*} d x=i \frac{4 \omega \mu_{0} l_{\delta}}{T} \sum_{n=-\infty}^{\infty} \frac{1}{k_{n}{ }^{3}} \frac{G_{n}(0)}{Q_{n}} C_{1 n} C_{2 n},
$$

где $C_{2 n}=\sum_{m=1}^{M} \dot{J}_{m} \sin \left(k_{n} \Delta_{m}\right) e^{-i k_{n} x_{m}} ; l_{\delta}$ - ширина индуктора по оси $y$.

Электромагнитная сила, действующая на обмотку индуктора в направлении движения, равна силе, действующей на расплав, и определяется выражением

$$
F_{e m}=\operatorname{Re}\left\{l_{\delta} \sum_{m=1}^{M} \int_{x_{m}-\Delta_{m}}^{x_{m}+\Delta_{m}} \dot{B}_{1 z}(x, 0) J_{m}^{*} \partial x\right\}=\operatorname{Re}\left\{i \frac{4 \mu_{0} l_{\delta}}{T} \sum_{n=-\infty}^{\infty} \frac{1}{k_{n}{ }^{2}} \frac{G_{n}(0)}{Q_{n}} C_{1 n} C_{2 n}\right\} .
$$

При взаимодействии магнитного поля с индуктированными в жидком металле токами возникают электромагнитные силы, средняя за период объемная плотность которых описывается выражением

$$
\bar{f}=\operatorname{Re}\left\{\dot{\bar{\delta}}_{2} \times \dot{\bar{B}}_{2}\right\}=\bar{e}_{x} f_{x}+\bar{e}_{z} f_{z}
$$

где

$$
f_{x}=\operatorname{Re}\left\{\dot{\delta}_{2 y} B_{2 z}^{*}\right\}, \quad f_{z}=-\operatorname{Re}\left\{\dot{\delta}_{2 y} B_{2 x}^{*}\right\} .
$$

Запишем выражения для относительных электромагнитной мощности и силы. За базисные мощность и силу примем величины, определяемые выражениями

$$
P_{b}=\frac{\omega \mu_{0} l_{\delta} 2 \tau^{2}}{\pi} J^{2} ; \quad F_{b}=\frac{P_{b}}{2 \tau \cdot f}=\mu_{0} l_{\delta} 2 \tau \cdot J^{2}
$$

где $J=\sum_{m=1}^{M} \frac{w_{m} I_{m}}{\tau}$

В соответствии с (34), (36), (39) и (40) будем иметь:

$$
\hat{S}_{e m}=\frac{S_{e m}}{P_{\delta}}=i \frac{\hat{T}^{2}}{4 \pi} \sum_{n=-\infty}^{\infty} \frac{1}{n^{3}} \frac{\hat{G}_{n}(0)}{\hat{Q}_{n}} \hat{C}_{1 n} \hat{C}_{2 n} ; \quad \hat{F}_{e m}=\frac{F_{e m}}{F_{\delta}}=\operatorname{Re}\left\{\frac{\hat{T}^{2}}{2 \pi} \sum_{n=-\infty}^{\infty} \frac{1}{n^{2}} \frac{\hat{G}_{n}(0)}{\hat{Q}_{n}} \hat{C}_{1 n} \hat{C}_{2 n}\right\},
$$

где $\hat{G}_{n}(0)=\hat{k}_{n} \cosh \left(k_{n}(z-\delta)\right)-\hat{\varphi}_{n} \sinh \left(k_{n}(z-\delta)\right) ; \hat{Q}_{n}=\hat{k}_{n} \sinh \left(k_{n} \delta\right)+\hat{\varphi}_{n} \cosh \left(k_{n} \delta\right) ;$

$$
\hat{\varphi}_{n}=\sqrt{\left(\frac{2 n}{\hat{T}}\right)^{2}+i \varepsilon\left(1-\frac{2 n}{\hat{T}}(1-s)\right)}
$$




$$
\begin{aligned}
& \hat{C}_{1 n}=\sum_{m=1}^{M} \hat{J}_{m} \sin \left(k_{n} \Delta_{m}\right) e^{-i k_{n} x_{m}} ; \hat{C}_{2 n}=\sum_{m=1}^{M} \hat{J}_{m}^{*} \sin \left(k_{n} \Delta_{m}\right) e^{i k_{n} x_{m}} ; \\
& \hat{k}_{n}=\frac{\tau}{\pi} k_{n}=\frac{2 n}{\hat{T}} ; \hat{T}=\frac{T}{\tau} ; \hat{J}_{m}=\frac{\dot{J}_{m}}{J} ; \hat{J}_{m}^{*}=\frac{J^{*}}{J} .
\end{aligned}
$$

Относительные активная и реактивная мощности соответственно таковы:

$$
\hat{P}_{e m}=\operatorname{Re}\left\{\hat{S}_{e m}\right\}, \quad \hat{Q}_{e m}=\operatorname{Im}\left\{\hat{S}_{e m}\right\} .
$$

Коэффициент мощности МГД-перемешивателя и электромагнитный коэффициент полезного действия устройства в двигательном режиме:

$$
\cos \varphi=\frac{\hat{P}_{e m}}{\sqrt{\hat{P}_{e m}{ }^{2}+\hat{Q}_{e m}{ }^{2}}} ; \quad \quad \eta_{e m}=\frac{\hat{F}_{e m}}{\hat{P}_{e m}}(1-s) .
$$

\section{Анализ расчета электромагнитных характеристик}

Как следует из полученных выражений, безразмерные характеристики индукционного МГД-перемешивателя определяют следующие параметры:

1. Количество пазов $M$, размеры пазов $2 \Delta_{m}$ и их расположение $x_{m}$, амплитудно-фазовые характеристики синусоидальных токов в пазах $\hat{\dot{J}}_{m}=\widehat{J}_{m} e^{i \psi_{m}}$, соотношение размеров индуктора $(L=2 p \tau=2 \tau)$ и ванны $(\widehat{T}=T / \tau)$.

2. Безразмерный коэффициент добротности $\varepsilon=\gamma \omega \mu_{0} \tau^{2} / \pi^{2}$.

3. Относительная величина зазора между индуктором и расплавом $-\delta / \tau$.

4. Скорость движения расплава (как твердого тела) относительно индуктора $-u$ или величина скольжения $s=1-u / \tau f$. Для упрощения сравнения характеристик будем полагать, что ширина всех пазов одинакова $\left(\Delta_{m}=c o n s t\right)$ и они занимают половину длинны индуктора, т.е. $\sum_{m=1}^{M} 2 \Delta_{m}=\tau$. Условие $T=2 \tau$ позволяет анализировать электромагнитное поле и электромагнитные характеристики без учета влияния продольного краевого эффекта. Рассмотрим три типа обмоток (рис. 3): трехфазная обмотка с фазной зоной $120^{\circ}$ (рис. $3 a$ ); двухфазная обмотка с фазной зоной $90^{\circ}$ (рис. 3б); трехфазная обмотка с фазной зоной $60^{\circ}$ (рис. 3в). Очевидно, что линейная токовая нагрузка во всех трех вариантах обмоток одинакова.



a

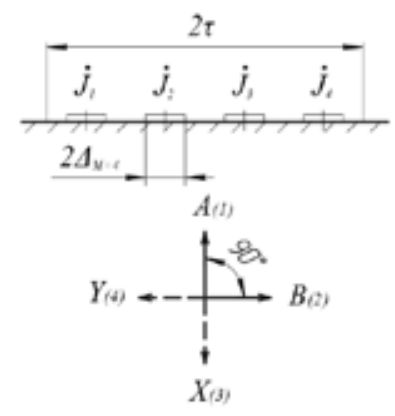

6

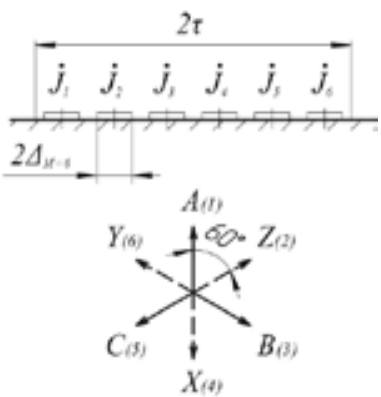

B

Рис. 3. Исследуемые типы обмоток индуктора 

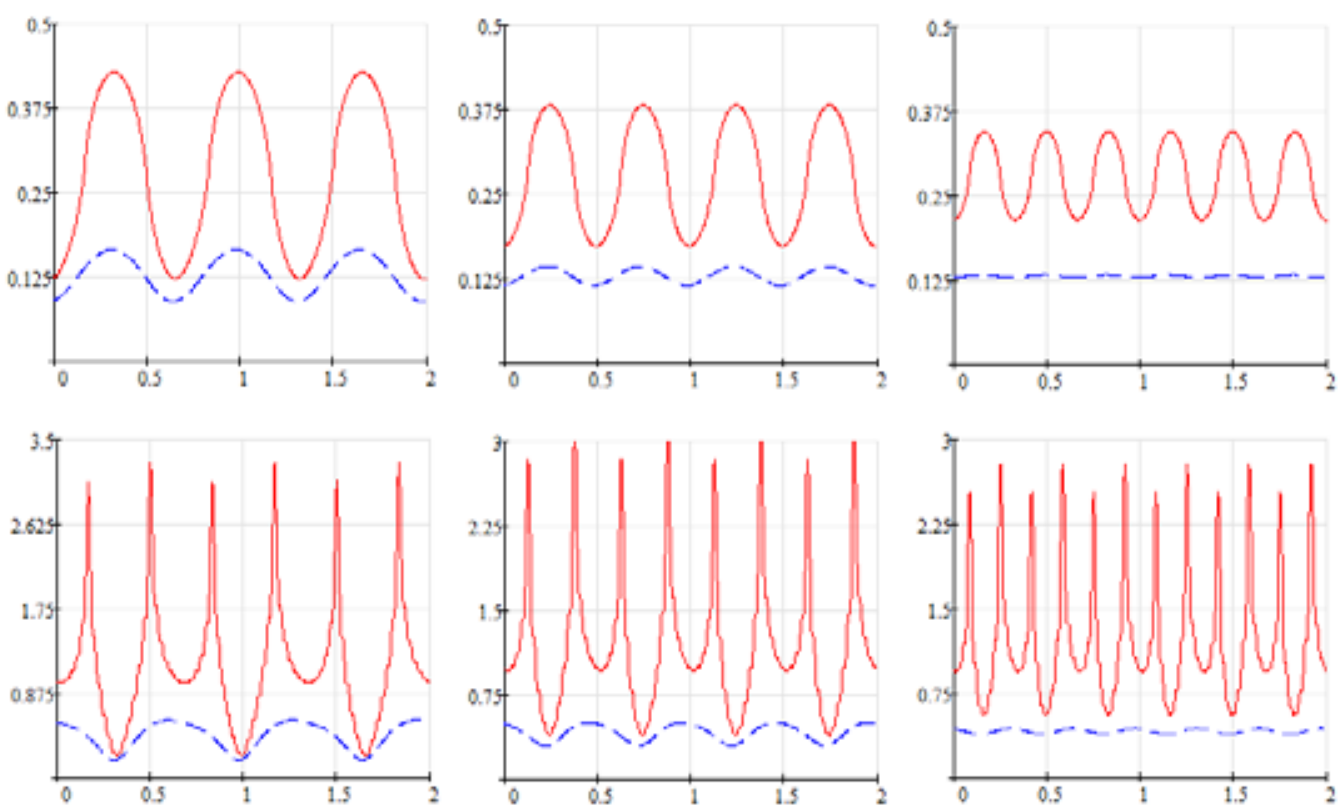

a

6

B

Рис. 4. Относительная напряженность электрического поля и нормальная составляющая относительной магнитной индукции для трех вариантов обмоток

На рис. 4 представлены огибающие кривые относительной напряженности электрического поля $\left(E_{1 y} / \omega \mu_{0} J \tau\right)$ и нормальной составляющей относительной магнитной индукции $\left(B_{1 z} / \mu_{0} J\right)$ на поверхности индуктора $(z=0)$ - сплошные линии - и поверхности расплава $\left(z=\delta^{\prime}\right)$ - пунктирные линии. По оси абсцисс показаны относительные значения. Кривые, представленные на рис. $4 a, 46,4 b$, соответствуют обмоткам на рис. $3 a, 3 \sigma, 36$. При расчете принималось $T=2 \tau$; $s=1 ; \delta / \tau=0,2 ; \varepsilon=2$, т.е. скорость расплава $u=0$. Если на поверхности индуктора зубцово-пазовые гармоники явно проявляются, то на поверхности расплава их проявление ослабевает. При этом с увеличением числа пазов проявление гармоник также уменьшается.

Картина распределения электромагнитного поля изменяется при наличии движения расплава относительно индуктора $(u \neq 0)$. На рис. 5 показаны те же огибающие при наличии движения. При расчете принималось $T=6 \tau ; s=0,1 ; \varepsilon=2 ; \delta / \tau=0,2$. От начала координат до входа в индуктор предусмотрено расстояние $\tau$, а от выхода индуктора до конца периода $T$ - расстояние $3 \tau$. Вследствие проявления продольного краевого эффекта на входе индуктора электромагнитное поле сжимается, а на выходе вытягивается. Неравномерность распределения электромагнитного поля по длине индуктора приводит к несимметрии токов в фазах при их подключении к источнику фиксированного напряжения. С уменьшением зазора $\delta$ неравномерность возрастает.

На рис. 6 и 7 представлены интегральные характеристики, соответствующие рассматриваемым обмоткам. Зависимости относительных активной $\hat{P}_{e m}$ и реактивной $\hat{Q}_{e m}$ мощностей от $\varepsilon$ при $\mathrm{M}=3,4,6$, показаны на рис. $6 a$ и $6 \sigma$ соответственно. На рис. 6 в показана зависимость $\cos \varphi=f(\varepsilon)$. 

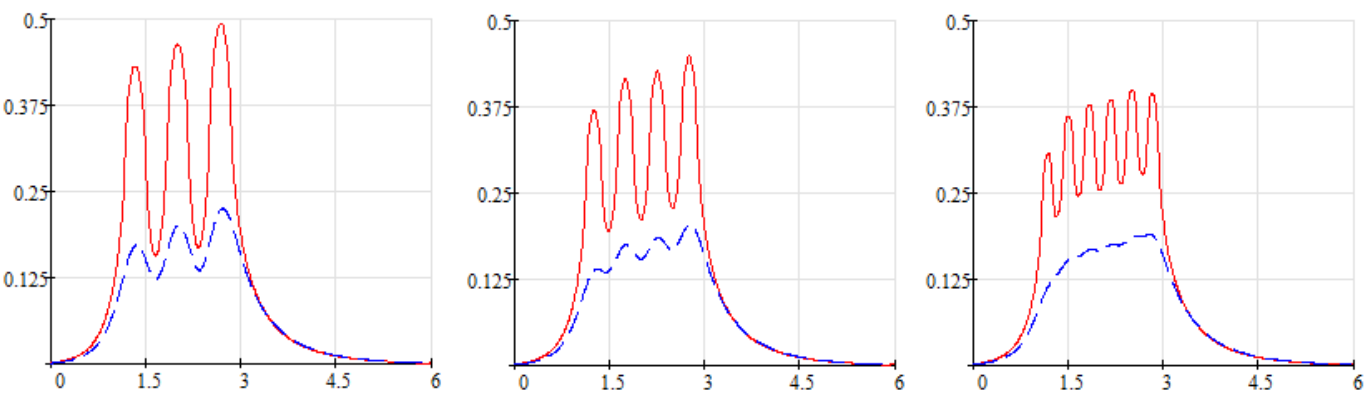

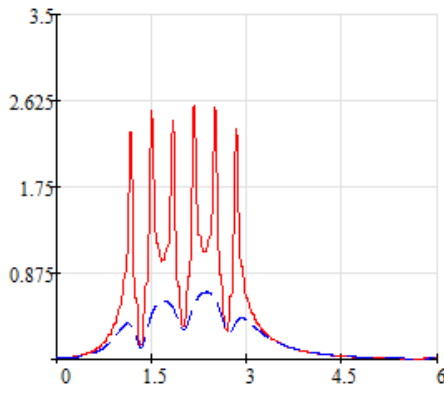

a

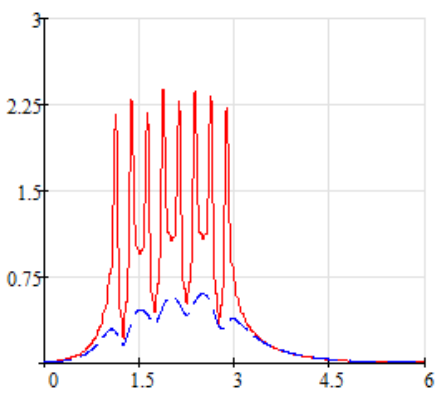

6

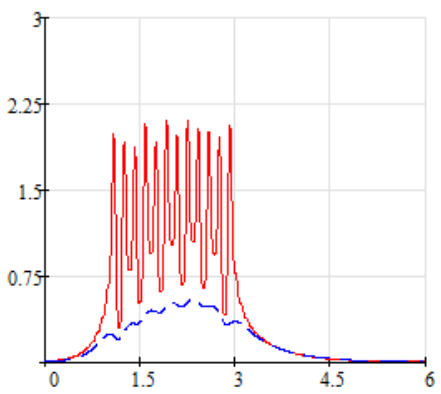

B

Рис. 5. Относительная напряженность электрического поля и нормальная составляющая относительной магнитной индукции для трех вариантов обмоток при $T \neq 2 \tau$ и $s=0,1$

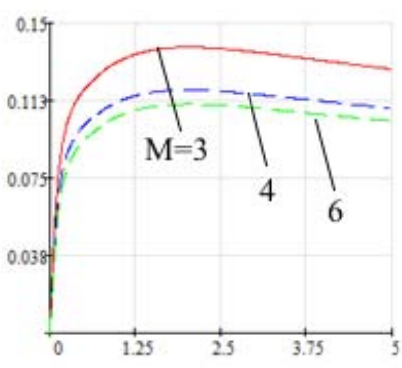

$\mathrm{a}$



6



B

Рис. 6. Относительные активная (а), реактивная мощности (б) и коэффициент мощности от $\varepsilon$ (в)

На рис. $7 a$ изображены зависимости относительной электромагнитной силы $\widehat{F}_{e m}$ от добротности $\varepsilon$, а на рис. $7 б$ представлены зависимости коэффициента полезного действия $\eta$ от скольжения $s$.

Как следует из представленных графиков, индуктор с трехфазной обмоткой с фазной зоной $120^{\circ}(M=3)$ потребляет из сети бо́льшие активную и реактивную мощности, чем индуктор с обмотками $M=4$ и 6 . Вместе с тем индуктор с этой обмоткой развивает электромагнитную силу $\widehat{F}_{e m}$, действующую на расплав, меньшую, чем индукторы с другими обмотками (рис. $7 a$ ). Этому соответствуют графики $\eta=f(s)$, представленные на рис. 76. С увеличением величины 

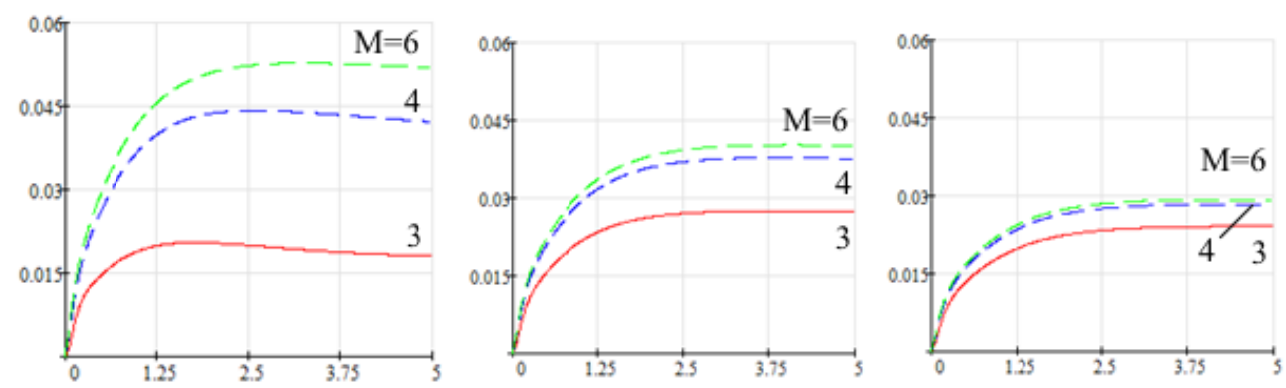

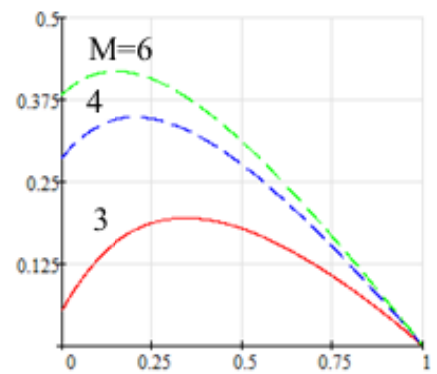

a

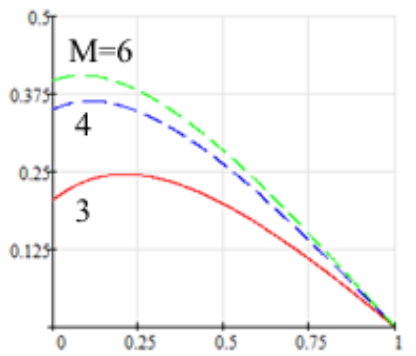

6

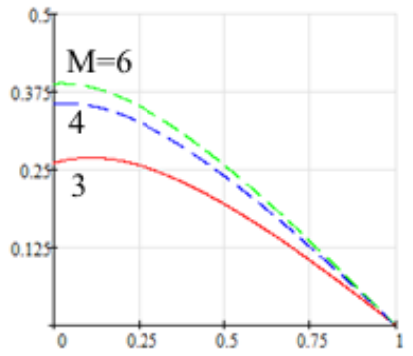

B)

Рис. 7. Относительная электромагнитная сила от $\varepsilon$ (сверху), КПД от $s$ (снизу): a $-\delta / \tau=0,2 ; 6-\delta / \tau=0,3$; B $-\delta / \tau=0,4$

зазора $(\delta / \tau$ от 0,2 до 0,4$)$ разность электромагнитных характеристик представленных обмоток уменьшается.

\section{Выводы}

На основе использования интегрального конечного преобразования с использованием рядом Фурье в комплексной форме решена двухмерная задача по анализу электромагнитных процессов в системе «МГД-перемешиватель - ванна с расплавом». Полученные выражения для дифференциальных характеристик: векторов электромагнитного поля, плотности тока и удельных электромагнитных сил в расплаве, позволяют анализировать гармонический состав электромагнитного поля в зазоре при различном дискретном распределении токовой нагрузки в индукторе и с учетом проявления продольного краевого эффекта. Выражения для электромагнитных мощности, силы, коэффициентов полезного действия и мощности дают возможность оценить требования к источнику питания МГД-перешивателя и эффективность его работы.

Проведен анализ дифференциальных и интегральных характеристик МГД-перешивателя с распространенными типами двухфазной и трехфазной обмотками, показаны их зависимости от коэффициента добротности и количества пазов индуктора.

Исследование выполнено при финансовой поддержке Российского фонда фундаментальных исследований, Правительства Красноярского края, Красноярского краевого фонда поддержки научной и научно-технической деятельности в рамках научных проектов № 16-48-242018 и № 16-43-242013 p_oфu_м. 


\section{Список литературы}

[1] Khatsayuk M, Timofeev V, Michaylov D, Research of MHD influence during preparation and casting aluminum melt in electromagnetic field, Applied Mechanics and Materials, 2015, 698, 181187.

[2] Baake E, Jakovics A, Khatsayuk M, Lupi S, Timofeyev V, MHD technologies in metallurgy. Intensive Course Specific IV. Study guide, Publishing house of ETU, St. Petersburg, 2013. 232.

[3] Вольдек А.И. Индукционные магнитогидродинамические машины с жидкометаллическим рабочим телом, Ленинград, Энергия, 1970. 272 c. [Vol'dek A.I. The induction MHD machines with liquid metal working fluid, Leningrad, Energy, 1970, 272 p. (in Russian)]

[4] Гринберг Г.А. Избранные вопросы математической теории электрических и магнитных явлений, Л.: Издательство АН СССР, Москва, 1948. 727 с. [Grinberg G.A. Selected problems of mathematical theory of electrical and magnetic phenomena. Moscow, USSR Academy of Sciences, 1948, 727 p. (in Russian)] 